\title{
Evaluation des Sols de Fondation de l'évacuateur de Crue du Petit Barrage de Youmban (Tillabéry-Niger)
}

\author{
Harouna Souley, \\ Ecole des Mines de l'Industrie et de la Géologie (EMIG) Niamey, Niger, \\ Département de Géologie, Faculté des Sciences et Techniques, \\ Université Abdou Moumouni, Niamey, Niger \\ Boureima Ousmane, \\ Département de Géologie, Faculté des Sciences et Techniques, \\ Université Abdou Moumouni, Niamey, Niger \\ Frédéric Collin, \\ Université de Liège, Département ArGenCo, \\ Laboratoire de Géotechnologies, Belgium
}

Doi: 10.19044/esj.2019.v15n6p434 URL:http://dx.doi.org/10.19044/esj.2019.v15n6p434

\section{Résumé}

Le barrage de Youmban se situe dans le canton de Gorouol, département de Téra (Région de Tillabéry, Niger). Il appartient à la zone semiaride du Liptako-Gourma. Ses coordonnées géographiques sont : 1448'7.80', de latitude $\mathrm{N}$ et $00^{\circ} 22^{\prime} 4.14^{\prime \prime}$ de longitude $\mathrm{E}$. Les apports d'eau à ce barrage, proviennent des deux grandes rivières temporaires localement connues sous les noms du Béli et du Gorouol. Depuis sa construction en 2004 ce barrage a connu une répétition de ruptures à l'emplacement de son organe d'évacuation de crue, dont la plus importante est celle intervenue au cours de la saison hivernale de 2011. Une réhabilitation proposée par le programme d'appui à la sécurité alimentaire (PASA) au courant de l'année 2012 a conduit à la construction d'un grand déversoir rigide en béton. La largeur déversante s'étend sur $250 \mathrm{~m}$ déduite de la crue centennale estimée à $287 \mathrm{~m}^{3} / \mathrm{s}$ (AC3E et al., 2012). Le béton étant un matériau qui ne supporte pas des efforts de traction, son choix en substitution aux ouvrages souples en gabions doit être justifié vis à vis des déformations du sol de fondation. C'est pourquoi la présente étude est initiée. Elle a pour objectif principal l'évaluation des caractéristiques géotechnique $\mathrm{du}$ sol de fondation. L'approche méthodologique est basée sur la réalisation des essais en place (essais au pénétromètre léger) et des essais au laboratoire (granulométrie, limites d'Atterberg, Proctor, cisaillement et odométrie). Des échantillons sont prélevés au moyen des puits manuels suivant l'axe de l'évacuateur. Ils sont numérotés $\mathrm{P} 1, \mathrm{P} 2$ et $\mathrm{P} 3$. Ainsi des résultats très intéressants ont été obtenus. 
Les sondages pénétrometriques montrent que les sols de fondation ont un taux de travail minimal de 2,8bars à partir de la profondeur de $2 \mathrm{~m}$. Ces sols sont soit des sols argileux sensibles à l'eau soit des sols alluvionnaires. L'analyse granulométrique corrobore ce résultat. En effet les échantillons P1, P2 et P3 testés, présentent plus de $35 \%$ de passants à $80 \mu \mathrm{m}$ avec un indice de plasticité supérieur à $11 \%$. Le poids volumique sec (essai Proctor) est sensiblement identique pour les échantillons P1 et P2 pour une valeur moyenne de $1,6 \mathrm{t} / \mathrm{m}^{3}$. L'échantillon P3 est beaucoup plus lourd avec une valeur de $1,88 \mathrm{t} / \mathrm{m}^{3}$ de poids volumique sec. Il est représentatif des dépôts alluvionnaires rencontrés au point de passage du cours d'eau Beli vers l'aval de la mare. Les essais de cisaillement direct ont donné une cohésion variant de $25 \mathrm{kPa}$ à $72 \mathrm{kPa}$ et un angle de frottement interne compris entre $9^{\circ}$ et $31^{\circ}$. Le calcul de stabilité intégrant ces valeurs montre que l'ouvrage est mécaniquement stable. Les essais de compressibilité réalisés, ont permis de calculer le tassement de la couche d'argile sous le poids du massif, une valeur de 6 à $21 \mathrm{~cm}$ a été trouvée. Les déformations obtenues sont relativement importantes, en effet il a été déduit une distorsion de l'ordre de 0,02 entre les panneaux de l'ouvrage en béton. Cette valeur est inadmissible pour un ouvrage qui régule la fonction de stockage d'eau (Skempton, 1952). En dépit de la solution qui consiste à construire un grand évacuateur en béton, il subsiste un risque d'exploitation de l'ouvrage lié au tassement de la fondation, ce qui peut compromettre sa sureté. Le barrage de Youmban impose donc un suivi et un entretien régulier de sa structure et de ses ouvrages annexes.

Mots-clés: Youmban, barrage, évacuateur, tassement, cohésion, Stabilité 


\title{
Evaluation of the Foundation Soils of the Spillway of the Small Youmban Dam (Tillabéry-Niger)
}

\author{
Harouna Souley, \\ Ecole des Mines de l'Industrie et de la Géologie (EMIG) Niamey, Niger, \\ Département de Géologie, Faculté des Sciences et Techniques, \\ Université Abdou Moumouni, Niamey, Niger
}

Boureima Ousmane,

Département de Géologie, Faculté des Sciences et Techniques,

Université Abdou Moumouni, Niamey, Niger

Frédéric Collin,

Université de Liège, Département ArGenCo, Laboratoire de Géotechnologies, Belgium

Abstract

The Youmban dam is located in Gorouol canton, Téra department (Tillabéry region, Niger). It belongs to the semi-arid zone of LiptakoGourma. Its geographical coordinates are: 14² $18^{\prime} 7.80^{\prime \prime} \mathrm{N}$ latitude and $00^{\circ} 22^{\prime} 4.14 " \mathrm{E}$ longitude. The water supply to this dam comes from the two large temporary rivers known locally as the Beli and Gorouol. Since its construction in 2004, this dam has experienced a repetition of ruptures at the site of its flood evacuation system, the most significant of which occurred during the 2011 winter season. a rehabilitation proposed by the food security support programme (PASA) in 2012 led to the construction of a large rigid concrete weir. The overhanging width extends over $250 \mathrm{~m}$ deducted from the 100-year flood estimated at 287m3/s (AC3E et al., 2012). Concrete being a material that does not support tensile forces, its choice as a substitute for flexible gabion structures must be justified with regard to deformations of the foundation ground. This is why this study is being initiated. Its main objective is to evaluate the geotechnical characteristics of the foundation soil. The methodological approach is based on the performance of existing tests (light penetrometer tests) and laboratory tests (particle size, Atterberg limits, Proctor, shear and oedometry). Samples are taken by means of manual wells along the axis of the spillway. They are numbered P1, P2 and P3. As a result, very interesting results have been obtained. Penetrometric surveys show that the foundation soils have a minimum working rate of $2.8 \mathrm{bar}$ from a depth of $2 \mathrm{~m}$. These soils are either clayey soils sensitive to water or alluvial soils. The granulometric analysis corroborates this result. Indeed, the samples P1, P2 and P3 tested, have more than $35 \%$ of passers-by at $80 \mu \mathrm{m}$ with a plasticity 
index higher than $11 \%$. The dry bulk density (Proctor test) is essentially identical for samples P1 and P2 with an average value of $1.6 \mathrm{t} / \mathrm{m} 3$. The P3 sample is much heavier with a value of $1.88 \mathrm{t} / \mathrm{m} 3$ dry bulk density. It is representative of the alluvial deposits encountered at the crossing point of the Beli river downstream of the pond. Direct shear tests gave a cohesion ranging from $25 \mathrm{kPa}$ to $72 \mathrm{kPa}$ and an internal friction angle between $9^{\circ}$ and $31^{\circ}$. The stability calculation incorporating these values shows that the structure is mechanically stable. The compressibility tests carried out made it possible to calculate the settlement of the clay layer under the weight of the massif, a value of 6 to $21 \mathrm{~cm}$ was found. The deformations obtained are relatively significant, in fact a distortion of about 0.02 has been deduced between the panels of the concrete structure. This value is not acceptable for a structure that regulates the water storage function (Skempton, 1952). Despite the solution of building a large concrete spillway, there is still a risk of operating the structure due to the settlement of the foundation, which can compromise its safety. The Youmban dam therefore requires regular monitoring and maintenance of its structure and associated structures.

Keywords: Youmban, dam, spillway, settlement, cohesion, Stability

\section{Introduction}

Dans le Liptako Gourma, les eaux souterraines sont très limitées et contenues dans des aquifères discontinus du socle cristallin et cristallophyllien (Ousmane B, 1988 ; Babayé, 2012). Aussi pour satisfaire leurs besoins en eau (eau domestique, eau d'irrigation, eau pour le bétail) les populations font recours aux eaux de surface provenant du Gorouol et de son sous-affluent le Beli. Malgré un contexte climatique difficile (évapotranspiration, étiage précoce, ensablement), il a été retenu la réalisation des petits barrages. Cependant ces ouvrages soufrent de beaucoup de désordres structurels compromettant les conditions de leur exploitation. C'est le cas du barrage de Youmban objet de cet article. La sureté de ce barrage est assurée par un organe d'évacuation de crue en béton fondé sur un sol argileux. Il s'agit d'évaluer les propriétés et caractéristiques de ce sol. Les objectifs spécifiques de l'étude sont les suivants :

- Réalisation des essais in situ à l'aide d'un pénétromètre dynamique léger ;

- Réalisation des essais de laboratoire portant sur l'identification (granulométrie et limites d'Atterberg) et la caractérisation mécanique (Cisaillement direct, œdomètre et Proctor).

Les essais de laboratoire sont effectués sur des échantillons prélevés à partir des sondages par puits manuels. Les points de sondages sont implantés le long de l'axe de l'évacuateur de crue. L'article comprend un aperçu sur les 
généralités quant à la zone d'étude, la méthodologie appliquée, les résultats obtenus et les discussions.

\section{Généralités sur la zone d'étude}

\section{Climat de la Zone d'Etude}

La zone d'étude se situe au Niger dans la région de Tillabéry précisément dans le Gourma. C'est une zone de transition entre le Sahara et le Sahel. Le climat de cette zone est aride. Il est caractérisé par des températures élevées $\left(41,3^{\circ} \mathrm{C}\right)$ et par l'alternance d'une longue saison sèche et d'une courte saison des pluies (MA, 2004). Les précipitations sont aléatoires mais elles permettent, en année favorable, le remplissage des mares. Les eaux de ces mares sont utilisées pour le développement de l'agriculture irriguée.

\section{Contexte hydrologique}

La délimitation schématique de la zone correspond à celle du bassin versant du Gorouol-Beli, cours d'eau alimentant le barrage. Elle est illustrée à travers la carte proposée par Gavaud (1967), modifiée dans le cadre de cette étude (Fig.1). Le Gorouol est le premier affluent du fleuve Niger à son entrée sur le territoire du Niger. Il suit une direction Est-Ouest, parcours sur lequel il traverse des zones marécageuses, des grandes mares et des dunes de sable avant de se jeter dans le fleuve Niger.

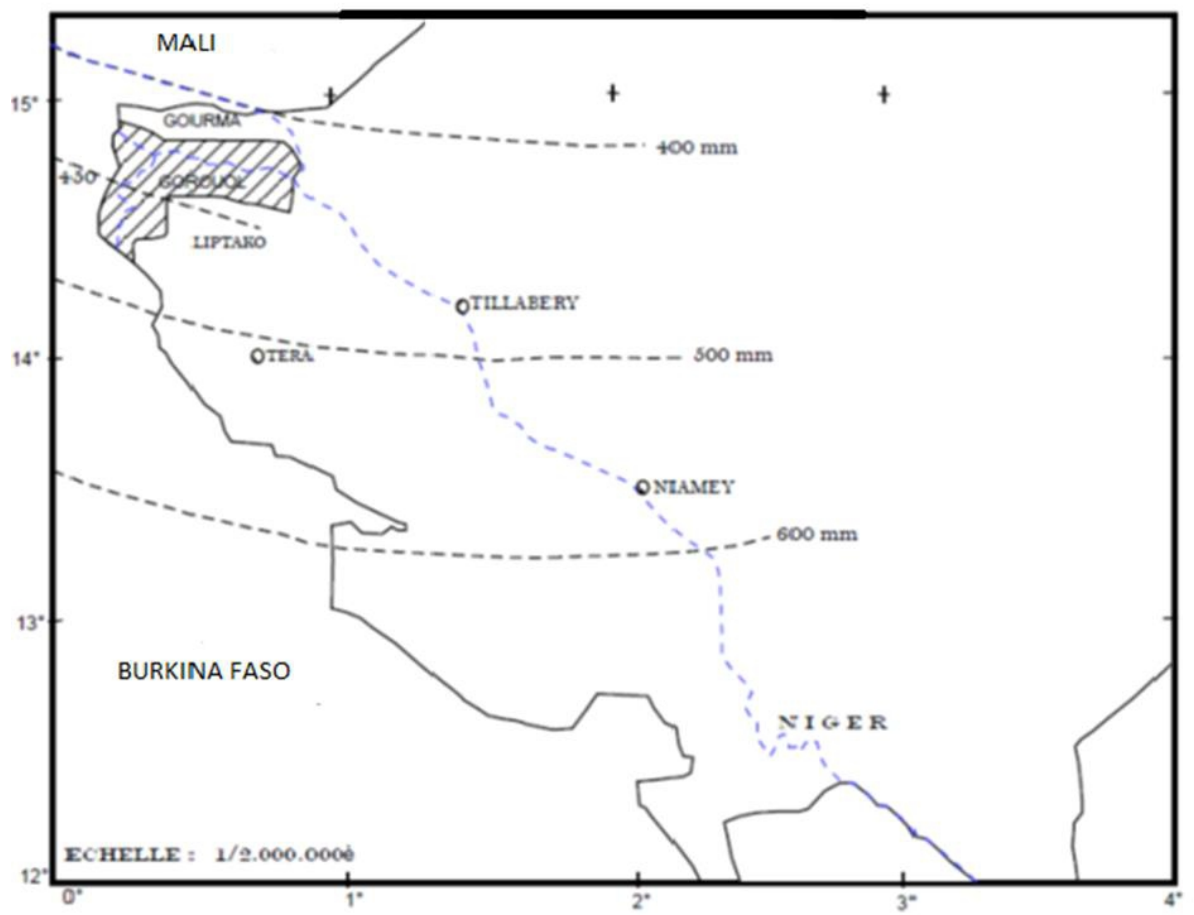

Figure 1: localisation du bassin du Gorouol (Gavaud, 1967 modifiée) et courbes des isohyètes de pluie. 
L'Office de la Recherche Scientifique et Technique d'Outre-mer (ORSTOM, 1970) a montré que de la succession de ces mares, résulte un effet d'amortissement des crues en amont du barrage. La plus importante des différentes mares traversées est la mare de Youmban aux coordonnées $00^{\circ} 46^{\prime} 13^{\prime \prime}$ et $00^{\circ} 22^{\prime} 37^{\prime \prime}$ de longitude Est et $14^{\circ} 46^{\prime} 13^{\prime \prime}$ et $14^{\circ} 49^{\prime} 10^{\prime \prime}$ de latitude Nord. C'est une gigantesque mare permanente (Fig.2) dont la superficie est de l'ordre de 525 hectares. Le tapis argileux structurant le fond de cette mare, a une conductivité hydraulique inférieure à $2,78.10^{-7} \mathrm{~m} / \mathrm{s}$ (Gavaud Y, 1967) ce qui la rend relativement étanche pour maintenir l'eau en permanence sur le cycle annuel.

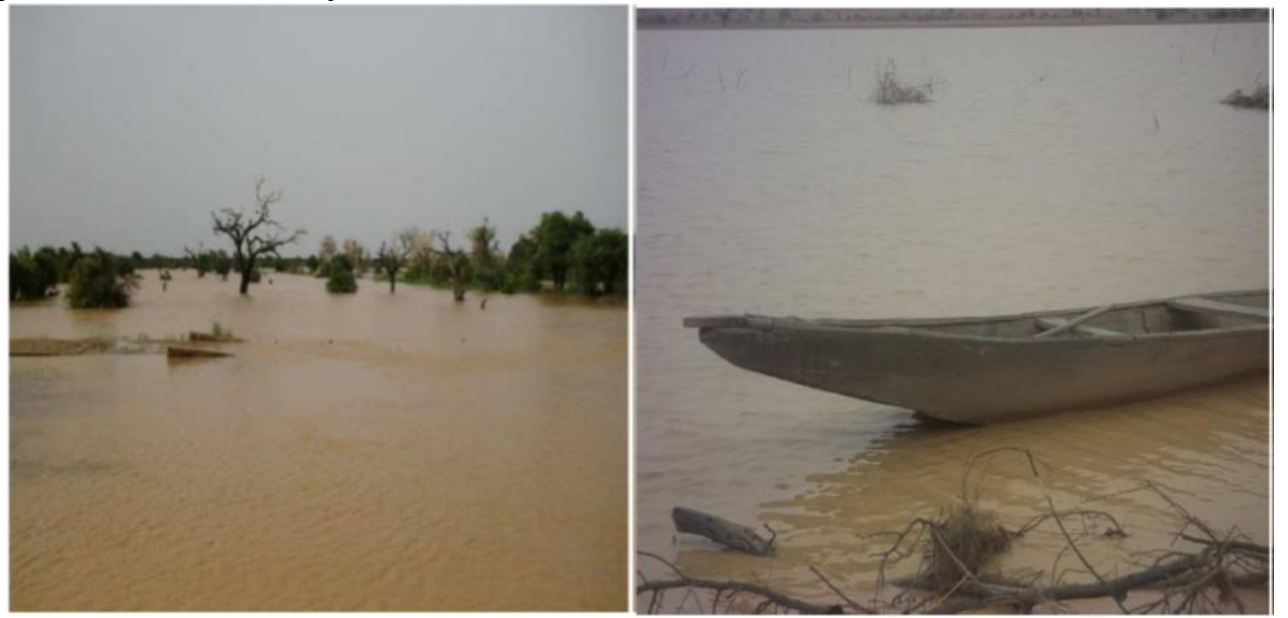

Figure 2: Photo de la retenue pleine de Youmban

Sur la base d'un échantillon de débit relevé à la station d'Alkongui, fourni par l'Autorité du Bassin du Niger (ABN, 2017), les apports annuels à la mare de Youmban sont estimés à 8,5 Millions de $\mathrm{m}^{3}$ en année normale. La crue centennale retenue pour le calcul de la largeur deversante de l'évacuateur des crues est de l'ordre de $287 \mathrm{~m}^{3} / \mathrm{s}$. Ce débit est largement supérieur à celui proposée par l'ORSTOM. Dans le cadre de l'étude hydrologique de la rivière Béli en provenance du Burkina Faso ORSTOM a en effet, trouvé une valeur de l'ordre de $100 \mathrm{~m}^{3} / \mathrm{s}$ (ORSTOM, 1970).

\section{Cadre géologique et hydrogéologique}

Les formations du Gourma se caractérisent par une altération rapide des schistes argileux, de grès, des quartzites de conglomérats, des calcaires ainsi que des calcaires dolomitiques. Les formations de couverture sont principalement constituées des dépôts éoliens structurés sous forme des cordons dunaires, des cuirasses latéritiques, des dépôts alluviaux (Babayé, 2012). L'étude spécifique du sol de Gorouol (Gavaud, 1967) a montré que le site abonde de matériaux ferrugineux tropicaux (latérites), de sols sableux et 
d'argiles. Ces formations sédimentaires (Fig.3) constituent les plus importants pourvoyeurs des géomatériaux dans cette zone.

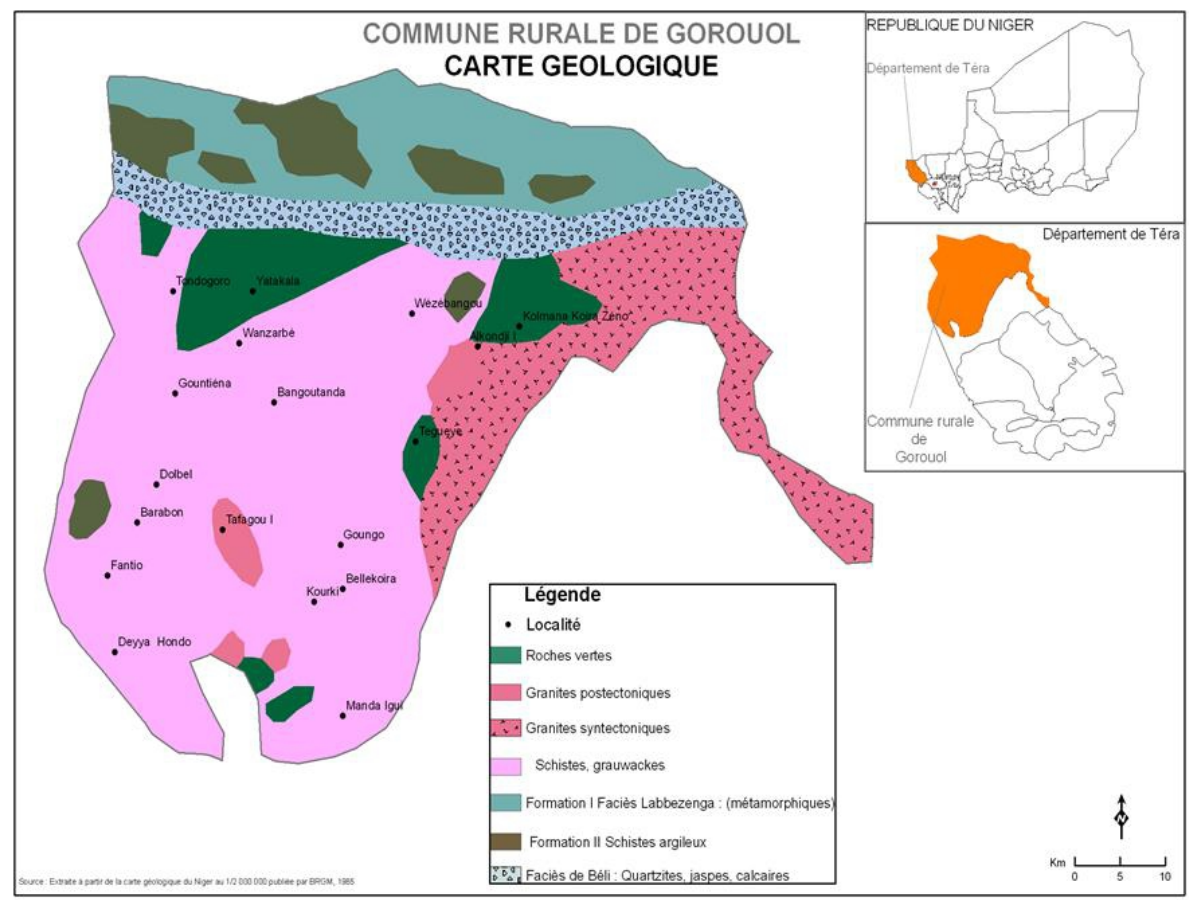

Figure 3: carte géologique de la commune du Gorouol modifiée (carte BRGM, 1967)

Sur le plan hydrogéologique les ressources en eau sont localisées dans les horizons fracturés fissurés et altérés, correspondant aux aquifères discontinus du socle (Ousmane B, 1988 ; Babayé, 2012). Dans les formations schisteuses du Gourma, les niveaux statiques sont situés entre 4,4 et 20,7 m. Les débits sont très faibles, ils varient entre 2,6 à $4,1 \mathrm{~m}^{3} / \mathrm{h}$.

\section{Caractéristiques du barrage de Youmban : La digue en terre}

Le barrage de Youmban comprend une digue homogène en terre argileuse et des organes d'évacuation de crue. Cette digue a été érigée sur le pourtour de la mare naturelle de Youmban. Elle épouse le contour incurvé de la mare qui s'étend sur une longueur d'environ $1,5 \mathrm{~km}$. Le profil général de la digue est de forme trapézoïdale. Sa hauteur est de l'ordre de $6 \mathrm{~m}$ à partir du fond de fondation. Sa largeur en crête est de $3 \mathrm{~m}$. Un rehaussement de la digue de $40 \mathrm{~cm}$ (Fig4) en matériaux latéritiques a été fait lors de la dernière réhabilitation en 2012.

La figure 3 montre également le talus amont et aval de la digue. La valeur du talus amont est de $2,5 \mathrm{H} / 1 \mathrm{~V}$, il est protégé au moyen d'un 
enrochement en vrac par endroit maçonné. Le talus aval présente une pente 2H/1 V, il est également protégé par des enrochements maçonnés.

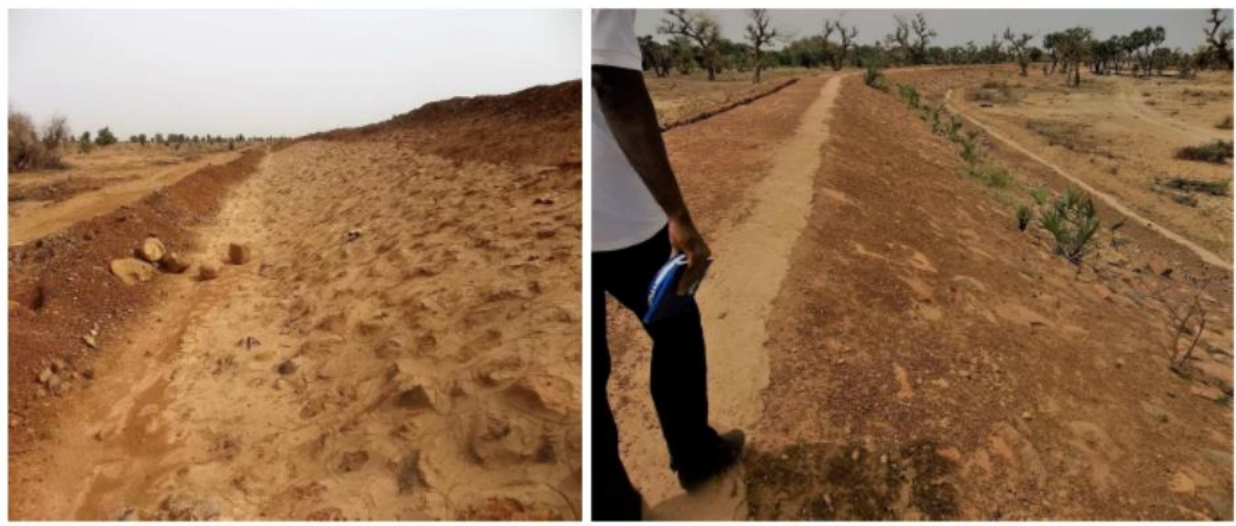

Figure 4: vues de la structure de la digue en amont et en aval.

\section{L'évacuateur de crue}

L'évacuateur de crue mis en place lors de la dernière réhabilitation de 2012(Fig.5) est un massif en béton à parement vertical conçu par tronçon de 5 mètres. Ces tronçons sont séparés des joints de dilatation de type caoutchouc «water-stop ». L'écoulement se fait par-dessus d'un déversoir mince. La restitution se fait par l'intermédiaire d'une cuillère imitant la forme classique du saut de ski. En pied du bloc est aménagé un bassin de dissipation en gabions L'emplacement de l'évacuateur est identique à celui choisi lors de la première construction de la digue, en rive gauche du Béli. La fouille de fondation (clef d'ancrage) a mis en évidence une couche argileuse sur plus de quatre mètres. Cette couche semble s'étendre beaucoup plus en profondeur au regard du caractère lithologique du bassin de Béli. Ce bassin présente une couche des schistes argileux sur plus de 300m (REICHEILT, R. 1971). 


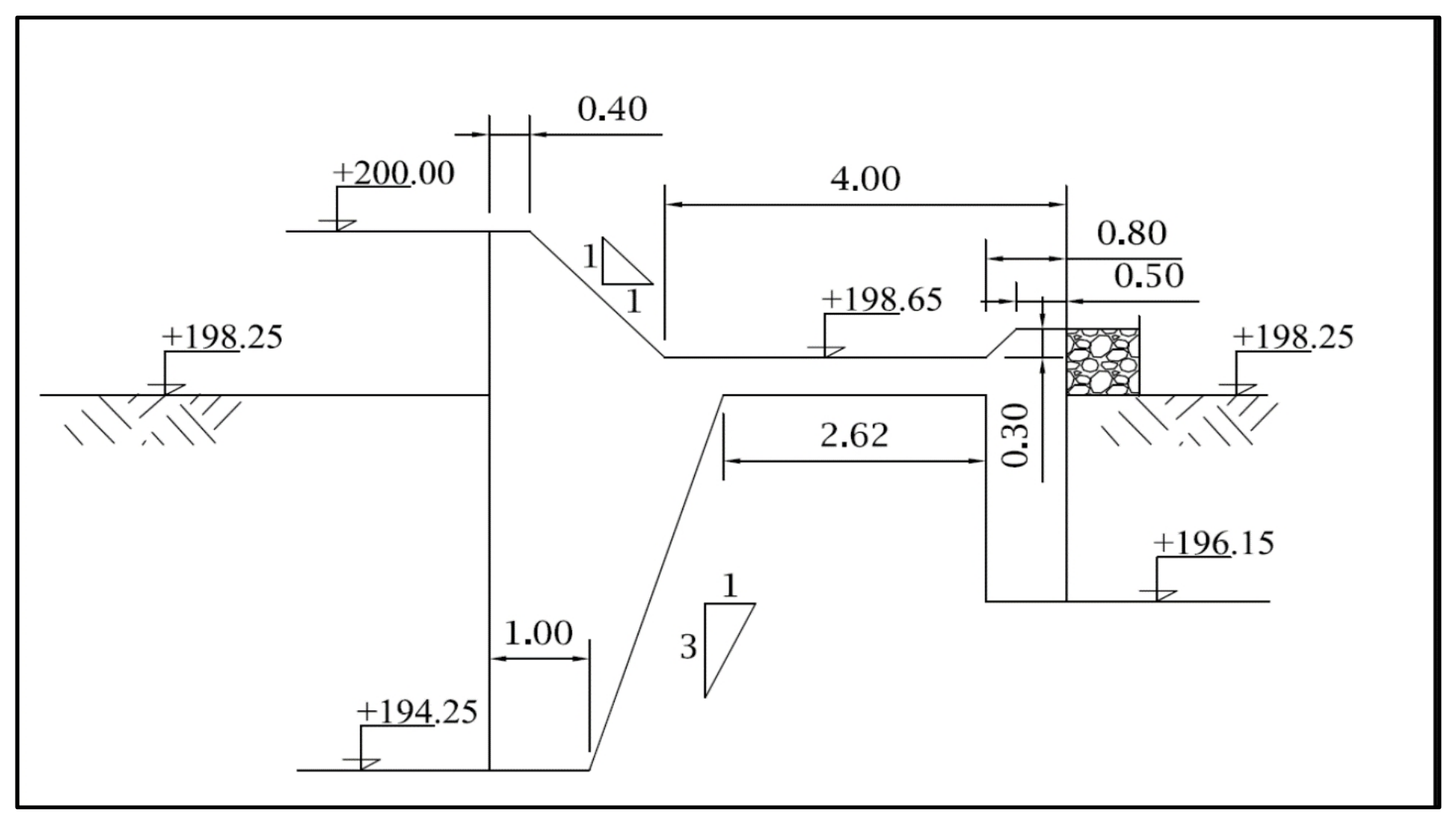

Figure 5 : Coupe du nouvel évacuateur de crue en béton. 


\section{Désordres observés}

Toutes les interventions sur le petit barrage de Youmban étaient liées aux problèmes d'évacuateur de crue. Cependant les désordres connus sur le barrage de Youmban ne peuvent être décrits que d'après les échanges avec les riverains. A titre illustratif, on ne dispose pas de documents écrits ou d'images de la crue qui avait dévasté le déversoir souple en gabion en 2010. La rupture de l'ouvrage était décrite par les riverains comme étant localisée entre la digue et le déversoir au niveau d'un ancien passage du Béli. Elle s'est traduite par l'affaissement et le basculement vers l'aval des ouvrages en gabions constituant la structure du déversoir. Les cages métalliques remplies de cailloux avaient été transportées sur une centaine de mètres à l'aval. Le scénario semble se répéter sur l'ouvrage en béton. En effet une année après sa construction, le déversoir rigide montrait des fissures profondes. Celles-ci sont visibles sur le mur bajoyer (Fig.6) avec une ouverture qui avoisinerait $2 \mathrm{~mm}$.

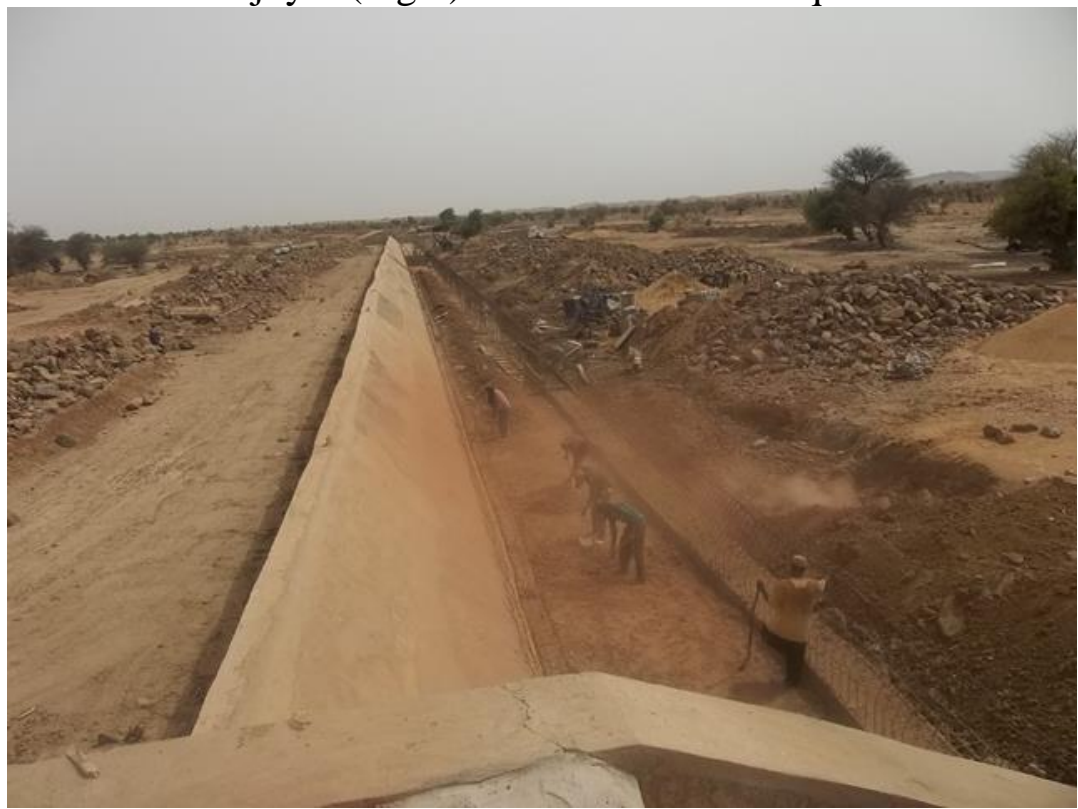

Figure 6: Evacuateur de crue en construction.

\section{Matériel et méthodes}

\section{Matériel:}

Le matériel utilisé pour la réalisation de l'étude, est constitué des outils et des données :

- Les outils de terrain: GPS GARMIN MAP 62, matériel de terrassement manuel (pelle, pioche, barre à mine) ; tarières manuelles ; pénétromètre dynamique léger T0013.1,

- Les outils de laboratoire : Tamis et appareils de Casagrande, Moules et dames Proctor, appareil de cisaillement direct et un œdomètre. 
- Les données: cartes; rapports d'étude des bases, les rapports techniques et les mémoires de thèses,-documents de classifications du Laboratoire Centrale des Ponts et Chaussées (LCPC) ainsi que les normes d'essai géotechnique (NF P94115 ; NF P 94-056 ; NF P 94051 ; NF P94-093 ; NF P94071-1, XP P94 090-1).

\section{Méthode:}

L'évaluation géotechnique objet de cet article a conduit à la proposition et l'exécution d'un programme de reconnaissance. Celui-ci comprend la réalisation des essais (au laboratoire et in situ) et le traitement des données. La consistance du programme de reconnaissance est la suivante :

- Une campagne de terrain ayant conduit à la réalisation des trois puits manuels $\left(\mathrm{P}_{1}, \mathrm{P}_{2}, \mathrm{P}_{3}\right)$ chaque $50 \mathrm{~m}$ le long de l'emplacement du déversoir. Ces puits permettront la description lithologique du sol;

- Une campagne de terrain par la réalisation de trois sondages (S1, S2 et S3) au moyen du pénétromètre dynamique léger en vue de déterminer la résistance dynamique en pointe des sols de fondation ;

- La réalisation des essais de laboratoire permettant l'identification et l'étude du comportement mécanique des sols. L'analyse granulométrique est réalisée sur les fractions argilo-sableuses rencontrées (selon norme NF P94 056). Tandis que la fraction argileuse est soumise aux essais de limites d'Atterberg (selon la norme NF P94 051). Vers les fonds des puits des échantillons sont prélevés pour l'essai de cisaillement et l'essai œdométrique. L'Essai Proctor est réalisé sur chaque sondage.

\section{Traitement des données :}

\section{Essai au pénétromètre léger:}

La résistance dynamique en pointe est calculée à partir de la formule des Hollandais développée dans le cas d'un choc élastique en négligeant les déformations du sol et du pénétromètre (Frazer, 1971 citée par Ben-Navarette, 2009). Cette formule s'écrit :

$R_{d}=\frac{M^{2} H}{A . e .(M+P)}$ où $\mathrm{Rd}$ est en $\mathrm{Kg} / \mathrm{cm}^{2} ; \mathbf{H}$ est la hauteur de chute en $\mathrm{cm} ;$ e est le refus (enfoncement par coup en $\mathrm{cm}$ ) ; M la masse du Mouton en $\mathrm{Kg}$; A l'aire de la section du cône en $\mathrm{cm}^{2} ; \mathbf{P}$ poids total tige\& enclume. Il est déduit la contrainte admissible $\left(\mathbf{q}_{\mathbf{c}}\right)$ d'après la relation proposée par Sanglerat (1971) : $q_{c}=\frac{R_{d}}{20}$.

\section{Essai de cisaillement:}

La résistance au cisaillement sera calculée d'après la théorie de rupture de Coulomb au moyen de la formule $\tau=C_{u}+\sigma \cdot \operatorname{tg} \varphi_{u}$ où $\tau$ désigne la contrainte 
tangentielle limite, $\sigma$ la contrainte normale appliquée, $\mathrm{C}_{\mathrm{u}}$ la cohésion non drainée et $\varphi_{u}$ l'angle de frottement non drainé. Les Courbes contraintes déformations sont obtenues d'après la procédure du Laboratoire Central des Ponts et Chaussées (LCPC, 1987).

\section{Essai œdomètre et évaluation du tassement:}

L'essai à l'œdomètre permet de connaitre les caractéristiques de déformabilité du sol de fondation. On peut par la suite calculer le tassement à long terme d'une couche de sol d'épaisseur $\mathrm{H}$ d'après la formule de Terzaghi. Cette formule est donnée par:

$$
\Delta H=C_{s} \frac{H}{1+e_{0}} \log _{10} \frac{\sigma_{c}^{\prime}}{\sigma_{0}^{\prime}}+C_{c} \frac{H}{1+e_{0}} \log _{10} \frac{\sigma_{0}^{\prime}+\Delta \sigma_{z}}{\sigma_{c}^{\prime}}
$$

Où : H est la hauteur initiale de l'échantillon; $\Delta H$, est le tassement observé; $e_{0}$ est l'indice des vides initial; $C_{c}$ est le coefficient de compressibilité, $C s$ est le coefficient de recompression, $\sigma_{0}^{\prime}$ est la contrainte effective due au poids des terres au-dessus du point à la cote z; $\sigma_{c}^{\prime}$ Contrainte de preconsolidation $=\sigma_{0}^{\prime}$ pour sol normalement consollidé, c'est la contrainte maximale appliquée et $\sigma_{0}^{\prime}+\Delta \sigma$ Contrainte finale appliquée au sol à la cote $\mathrm{z}$.

\section{Calcul de stabilité de l'ouvrage}

Ce paragraphe est relatif à la vérification successivement des stabilités au renversement, au glissement et au poinçonnement de l'évacuateur. La stabilité au renversement consisterait à l'étude de l'équilibre entre le moment des forces qui tendent à maintenir le bloc dans sa position et celui des forces qui cherchent à le renverser. Les actions considérées sont :

- La pression hydrostatique donnée par la formule $P=\frac{\gamma_{\omega} H^{2}}{2}+\gamma_{\omega} H . h$; $\gamma_{\omega}$ est le poids volumique de l'eau pris à $10 \mathrm{kN} / \mathrm{m}^{3}$.

- la poussée des sédiments qui s'accumulent souvent au pied amont du bloc en béton. Elle s'exprime par la formule : $P_{T}=\frac{1}{2} \gamma_{i} h^{\prime 2} \operatorname{tg}^{2}\left(\frac{\pi}{4}-\frac{\varphi}{2}\right)$; où $h$ ' est la hauteur des sédiments, l'angle de frottement à approximativement une valeur de $20^{\circ}$

- Le poids du massif en béton avec l'hypothèse d'un poids volumique de $24 \mathrm{kN} / \mathrm{m}^{3}$;

- La pression interstitielle varie linéairement de la valeur $\gamma_{\omega} H$ en amont à la valeur $\gamma_{\omega} h$ en aval.

Le critère de stabilité au renversement est que le facteur de sécurité obtenu en faisant le rapport des moments des forces stabilisatrices sur les moments des forces déstabilisatrices considérés par rapport à son pieds aval est supérieur à $\mathbf{1 , 5}$. 
Quant au calcul de stabilité au glissement, la composante tangentielle de glissement admissible sous le bloc est comparée à la composante tangentielle réellement appliquée. La composante tangentielle admissible correspond à celle obtenue d'après l'équilibre de Coulomb : $T_{a d m}=C_{u} . B+$ $N \operatorname{tg} \delta$ ou $\mathrm{C}_{\mathrm{u}}$ désigne la cohésion non drainée du sol obtenue par l'essai de cisaillement, B la largeur à la base du Bloc évacuateur en mètres, $\mathrm{N}$ les forces normales à la base, $\delta$ l'angle de frottement sol-Mur. On doit vérifier que la force admissible rapportée aux efforts tangentiels donne un rapport inférieur à 1,5 .

L'étude de stabilité au poinçonnement consiste à vérifier que sous le mur la pression normale maximum est toujours inferieure à la contrainte admissible $\left(\mathrm{q}_{\mathrm{adm}}\right)$ où $\mathrm{q}_{\mathrm{adm}}$ est donnée par:

\section{Résultats}

\section{Les puits manuels}

Les coupes des puits (Fig.7) laissent apparaitre une couche uniforme d'argile tout le long des profils P1 et P2. Ceux-ci sont situés respectivement en rive droite et en position centrale le long de l'axe de l'évacuateur. Vers la rive gauche, l'argile a un caractère sableux (puits $\mathrm{P} 3$ ). Au point $\mathrm{P} 1$, le sondage est arrêté à $2 \mathrm{~m}$, du fait de la présence d'une couche d'argile très compacte, difficile à extraire manuellement. Le puits P3 est localisé sur l'ancien point de passage du Béli avant la réalisation du barrage. Ceci peut expliquer la présence des dépôts alluvionnaires sableux observés en ce point. Ces dépôts constitueraient une structure apparente très différente de la structure originale des terrains obtenue en P1 et P2. La singularité du point P3 corrobore les ruptures répétées de l'évacuateur de crue du barrage en ce point.

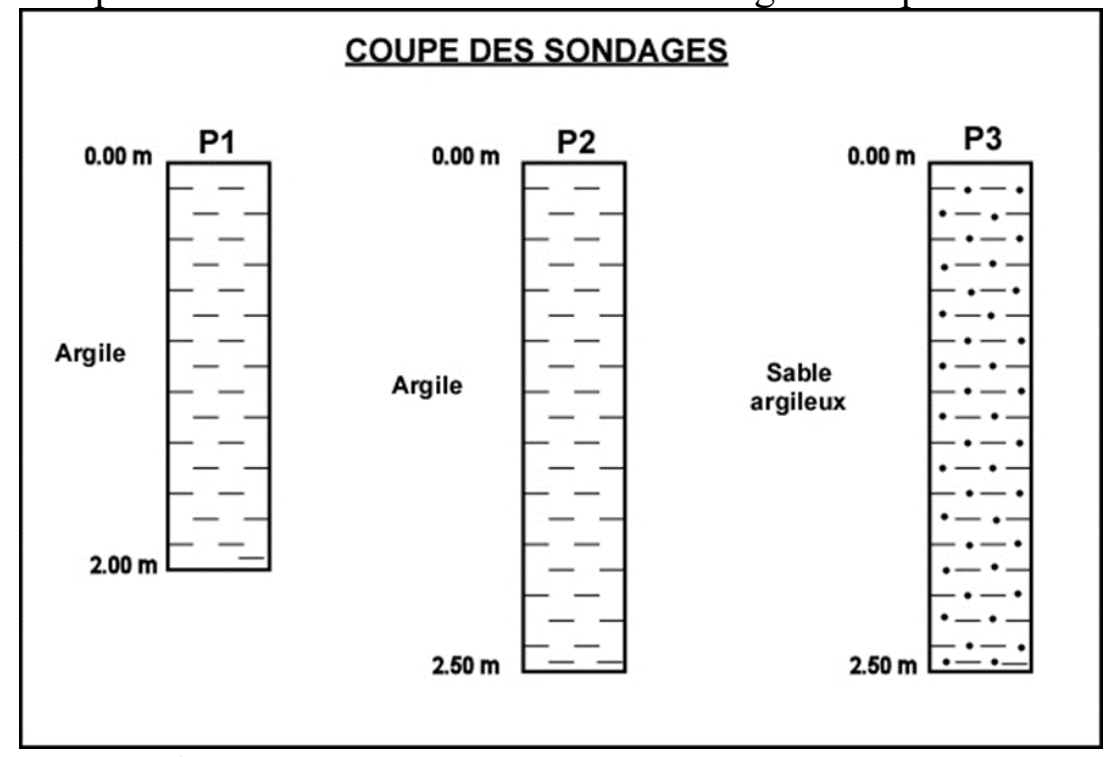

Figure 7:coupes lithostratigraphiques des puits P1, P2, P3 


\section{Sondages au pénétromètre léger:}

Afin de déterminer la portance des sols, trois sondages pénétrometriques $\mathrm{S} 1, \mathrm{~S} 2$ et $\mathrm{S} 3$ ont été réalisés presque dans les mêmes positions que les puits $\mathrm{P} 1, \mathrm{P} 2$ et $\mathrm{P} 3$. Les résultats obtenus sont représentés sur la figure 8 . Ces sondages ont été exécutés à des profondeurs variables en fonction du refus au point. La profondeur maximale de sondage a atteint $4 \mathrm{~m}$ au point S3. Aux points S2 et S1 il n'a été atteint respectivement, que 3,5m et $2,5 \mathrm{~m}$ de profondeur. La figure 7 montre qu'à 2 mètres de profondeur, le sol en S1 présente la plus faible valeur de la résistance dynamique en pointe $(\mathrm{RDP}=56 \mathrm{bars})$. Des valeurs plus importantes sont obtenues à cette même profondeur au sondage $\mathrm{S} 2$ ( $\mathrm{RDP}=74 \mathrm{bars}$ ) et au sondage $\mathrm{S} 3$ ( $\mathrm{RDP}=124 \mathrm{bars}$ ).

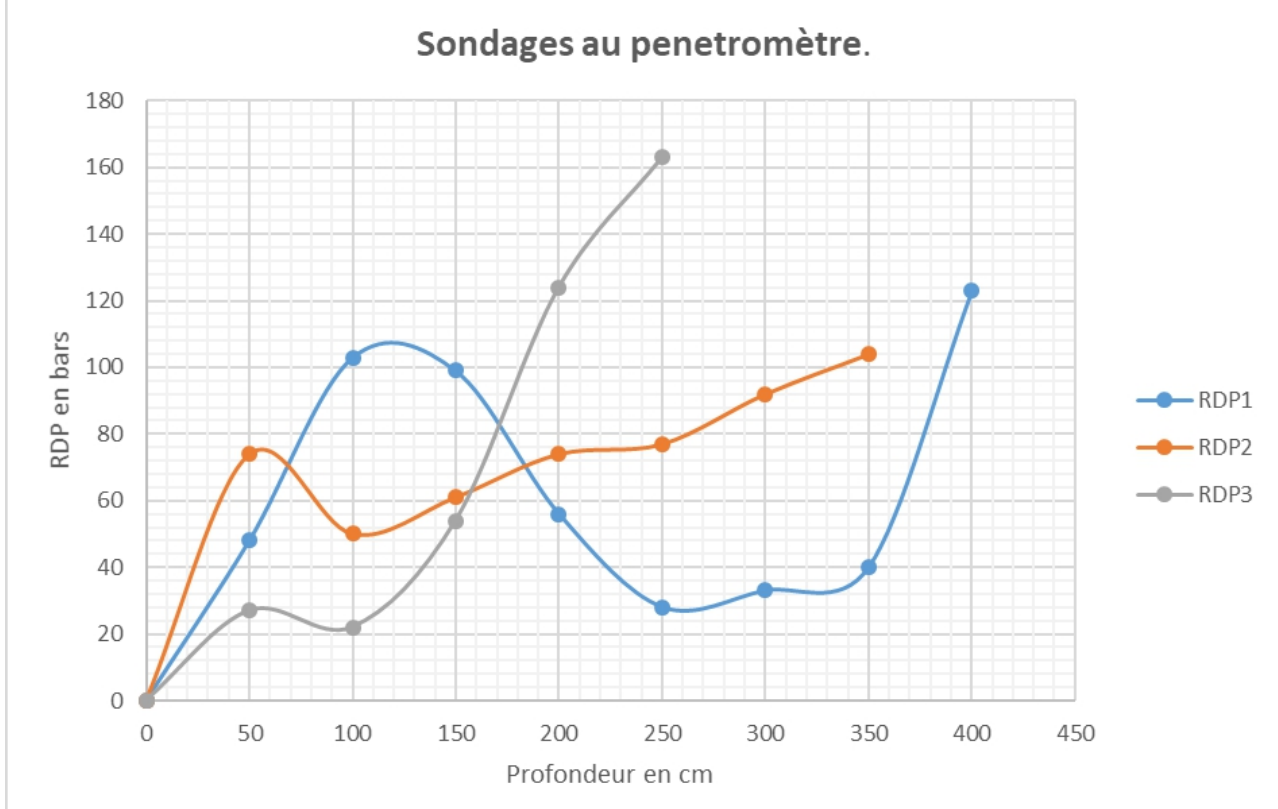

Figure 8: Courbes de sondage au pénétromètre dynamique léger.

Le tableau 1 donne les valeurs des contraintes admissibles déduites des résistances dynamiques en pointe.

Tableau 1: valeurs comparés de Rd et qc en bars

\begin{tabular}{|c|c|c|c|c|c|c|}
\hline \multirow{2}{*}{ Profondeur en cm. } & \multicolumn{2}{|c|}{ S1 } & \multicolumn{2}{c|}{ S2 } & \multicolumn{2}{c|}{ S3 } \\
\cline { 2 - 7 } & $\mathrm{Rd}$ & $\mathrm{qc}$ & $\mathrm{Rd}$ & $\mathrm{qc}$ & $\mathrm{Rd}$ & $\mathrm{Qc}$ \\
\hline 50 & 48 & 2,4 & 74 & 3,7 & 27 & 1,35 \\
\hline 100 & 103 & 5,15 & 50 & 2,5 & 22 & 1,1 \\
\hline 150 & 103 & 5,15 & 61 & 3,05 & 54 & 2,7 \\
\hline 200 & 56 & $\mathbf{2 , 8}$ & 74 & 3,7 & 124 & $\mathbf{6 , 2}$ \\
\hline 300 & 33 & 1,65 & 92 & 4,6 & - & - \\
\hline 400 & 123 & 6,15 & 104 & 5,2 & - & - \\
\hline
\end{tabular}




\section{Essais de laboratoire}

Les résultats obtenus sont rassemblés ci-dessous avant de retrouver les éléments d'interprétation et de discussion dans la partie « Discussion ».

\section{Analyse granulométrique et limites d'Atterberg}

Les courbes granulométriques (Fig.9) donnent la répartition dimensionnelle des échantillons. L'identification des échantillons a été complétée par les essais de détermination des limites d'Atterberg. Le tableau 2 indique les pourcentages des éléments passants à $0,08 \mathrm{~mm}$, à $0,5 \mathrm{~mm}$ et à $2 \mathrm{~mm}$ ainsi que la limite de liquidité et l'indice de plasticité pour chaque échantillon.

Tableau 2 : Caractéristiques granulométriques et valeurs des limites d'Atterberg.

\begin{tabular}{|c|c|c|c|c|c|}
\hline \multirow{2}{*}{ Echantillon } & \multicolumn{3}{|c|}{ Analyse granulométrique } & \multicolumn{2}{c|}{ Limites d'Atterberg } \\
\cline { 2 - 6 } & $\%<80 \mu \mathrm{m}$ & $\%<0,5 \mathrm{~mm}$ & $\%<2 \mathrm{~mm}$ & Wl en \% & Ip en \% \\
\hline P1 & 93 & 99 & 100 & 36,50 & 16 \\
\hline P2 & 74 & 97 & 100 & 58,2 & 27,8 \\
\hline P3 & 47 & 95 & 99 & 23,3 & 11 \\
\hline
\end{tabular}

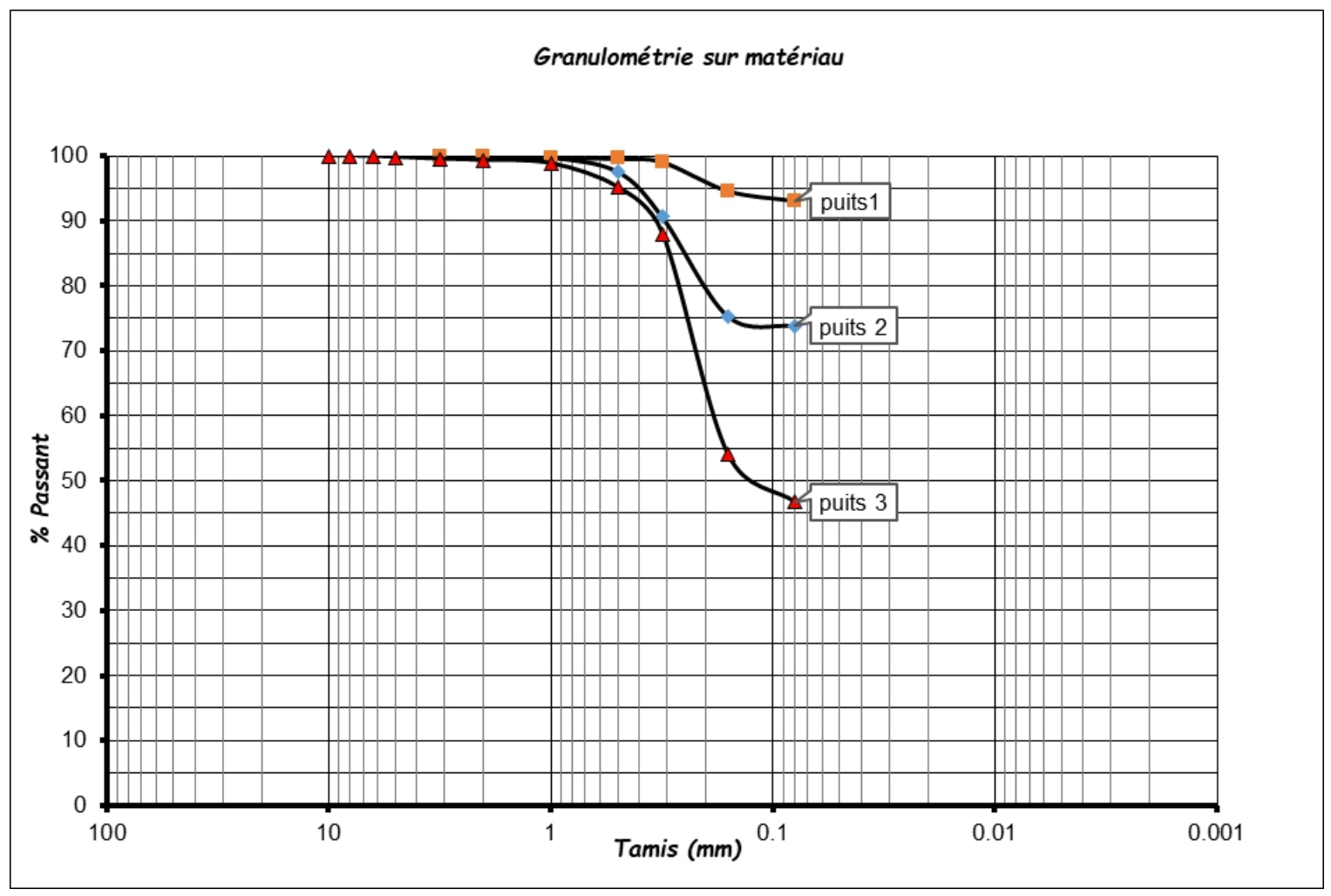

Figure 9: Courbes granulométries des échantillons issus de P1, P2, P3. 
Tenant compte des valeurs de référence de la classification P1, P2 et P3 sont des échantillons pour lesquels on note plus de $35 \%$ de passants à $80 \mu \mathrm{m}$ avec un indice de plasticité supérieur à 11\% (Fig.9).

\section{Essais Proctor:}

La détermination des caractéristiques de compactage des échantillons a été réalisée au moyen des essais Proctor modifié dont les caractéristiques à l'optimum sont rassemblées dans le tableau 3.

Tableau 3 : résultats essais Proctor

\begin{tabular}{|c|c|c|}
\hline \multirow{2}{*}{ Echantillon } & \multicolumn{2}{|c|}{ Caractéristiques Proctor } \\
\cline { 2 - 3 } & Teneur en eau optimale en \% & Poids volumique sec en $\mathrm{t} / \mathrm{m}^{3}$ \\
\hline P1 & 17,90 & 1,64 \\
\hline P2 & 21,6 & 1,57 \\
\hline P3 & 10,40 & 1,88 \\
\hline
\end{tabular}

\section{Cisaillement Direct:}

Des cisaillements directs avaient été réalisés sur des échantillons remaniés reconstitués tenant compte des résultats obtenus à l'essai Proctor, puis consolidés pendant 24 heures. Les grandeurs mesurées sont la cohésion (C) et l'angle de frottement interne $(\varphi)$ en condition non drainée (Fig. 10, 11,12).

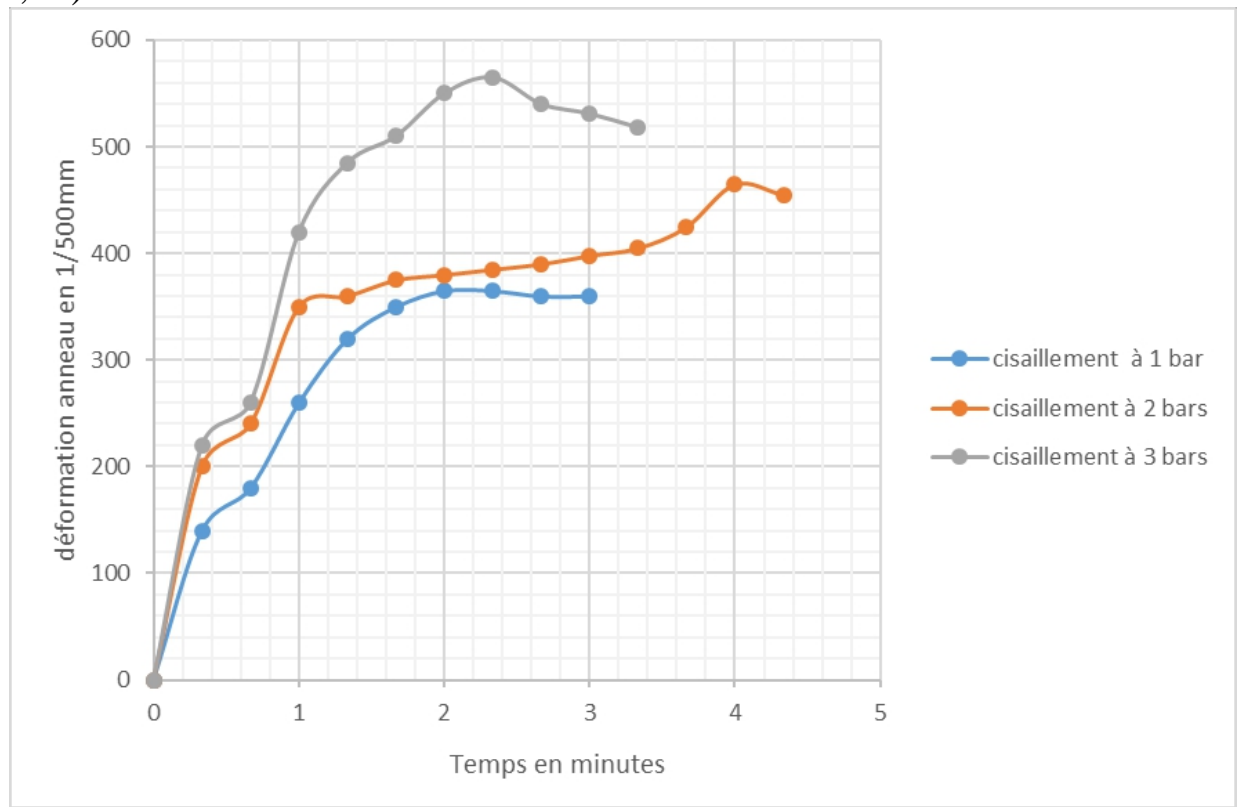

Figure 10 : Courbes de cisaillement au point P1. 


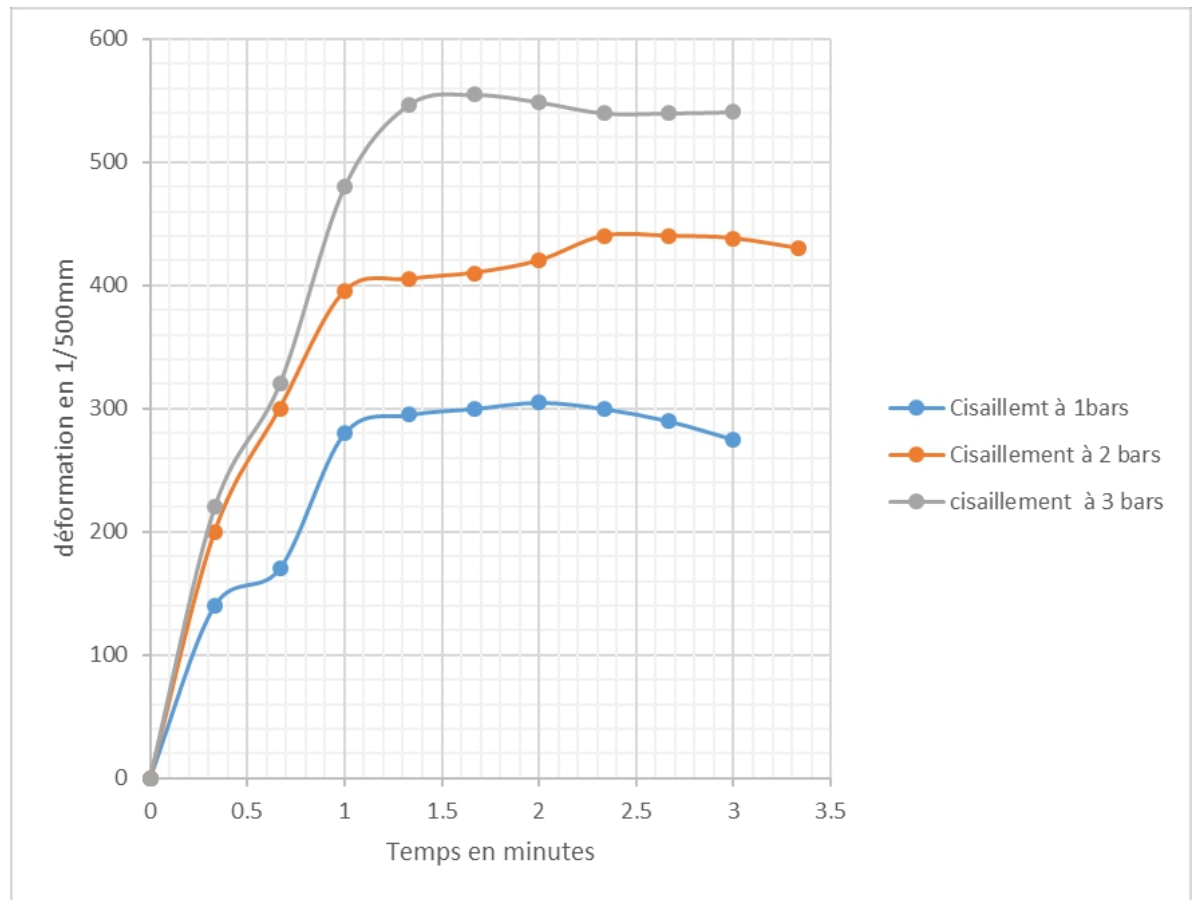

Figure 11: Courbes de cisaillements au point P2.

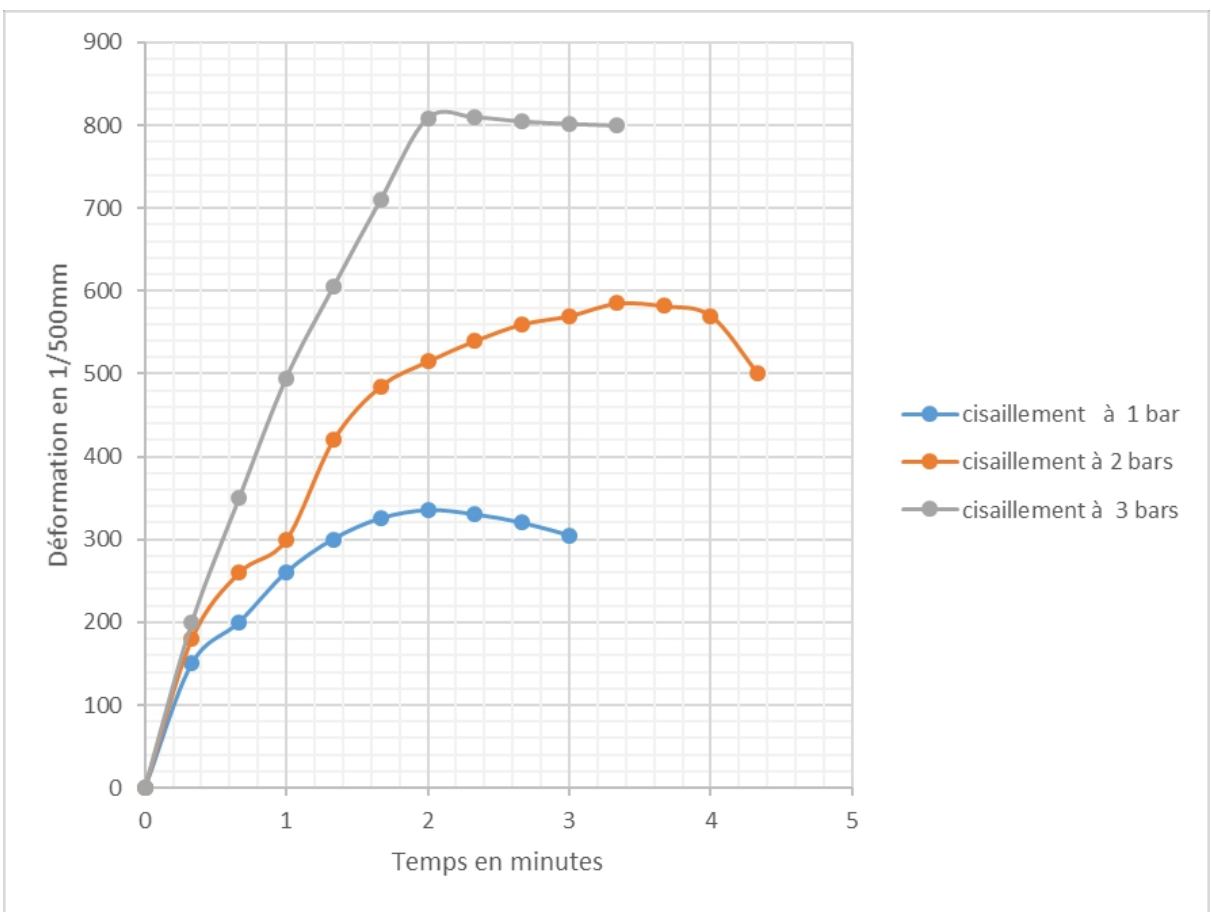

Figure 12: Courbes de cisaillement au point $\mathrm{P} 3$. 
La synthèse des caractéristiques mécaniques $\mathrm{C}_{\mathrm{u}}$ et $\varphi_{u}$ est donnée dans le tableau 4.

Tableau 4 : Essais sur les échantillons P1, P2, P3.

\begin{tabular}{|c|c|c|}
\hline \multirow{2}{*}{ Echantillons } & \multicolumn{2}{|c|}{ Caractéristiques mécaniques. } \\
\cline { 2 - 3 } & $\mathrm{C}_{\mathrm{u}}$ en $\mathrm{kPa}$ & $\varphi_{\mathrm{u}}$ en degré \\
\hline $\mathrm{P} 1$ & 72,33 & 9 \\
\hline $\mathrm{P} 2$ & 50 & 16 \\
\hline $\mathrm{P} 3$ & 25 & 31 \\
\hline
\end{tabular}

\section{Essais œedométriques}

Les essais œdométriques réalisés sur les échantillons P1, P2 et P3 prélevés au fond des puits manuels, ont permis de tracer les courbes de compressibilité (Fig.13, 14 et 15) . Les échantillons P1, P2 et P3 sont initialement partiellement saturés. Leurs degrés de saturation sont respectivement évalués à $23,9 \%, 27,4 \%$ et $14,8 \%$.

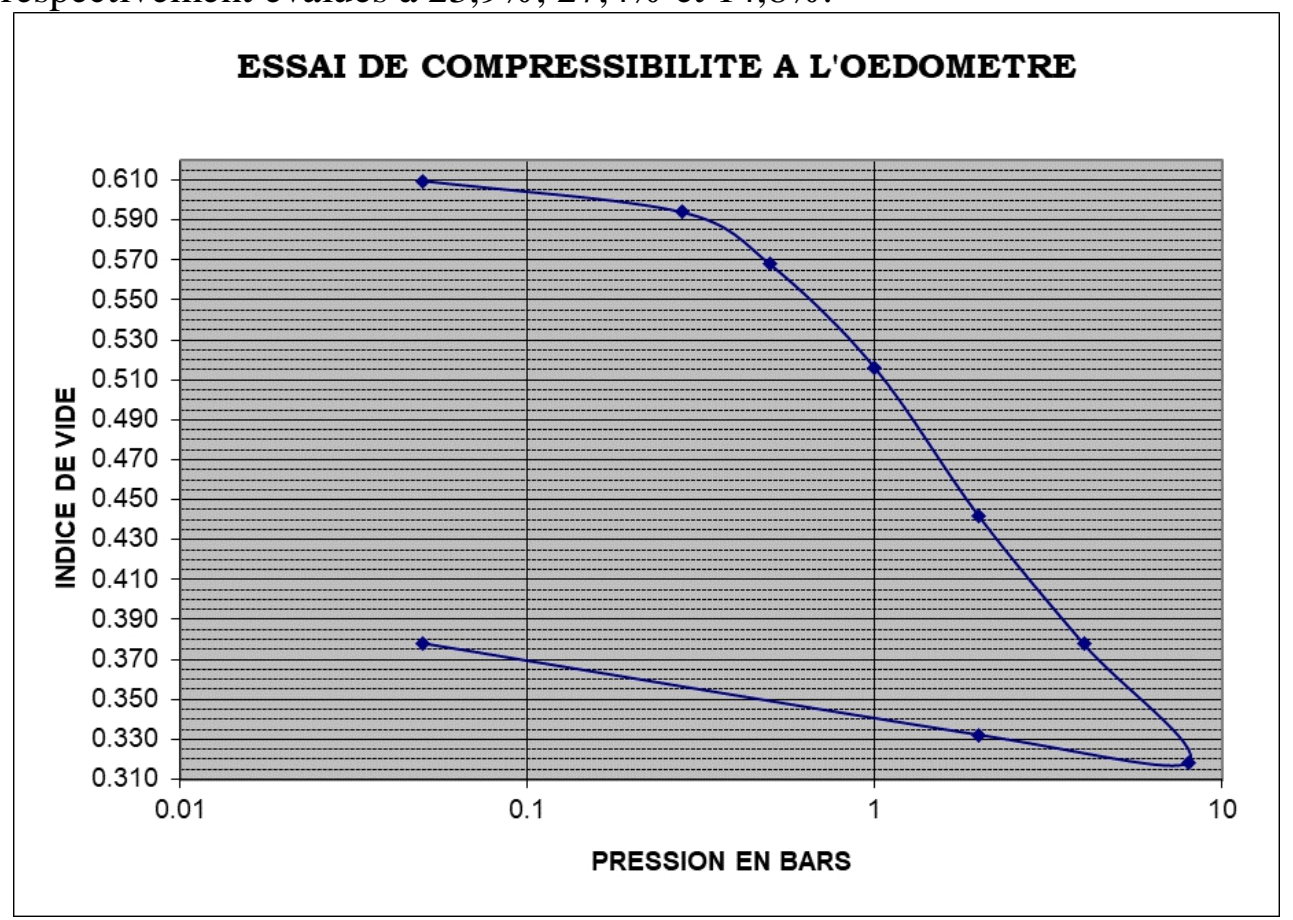

Figure 13: courbe de compressibilité à l'odomètre en P1. 


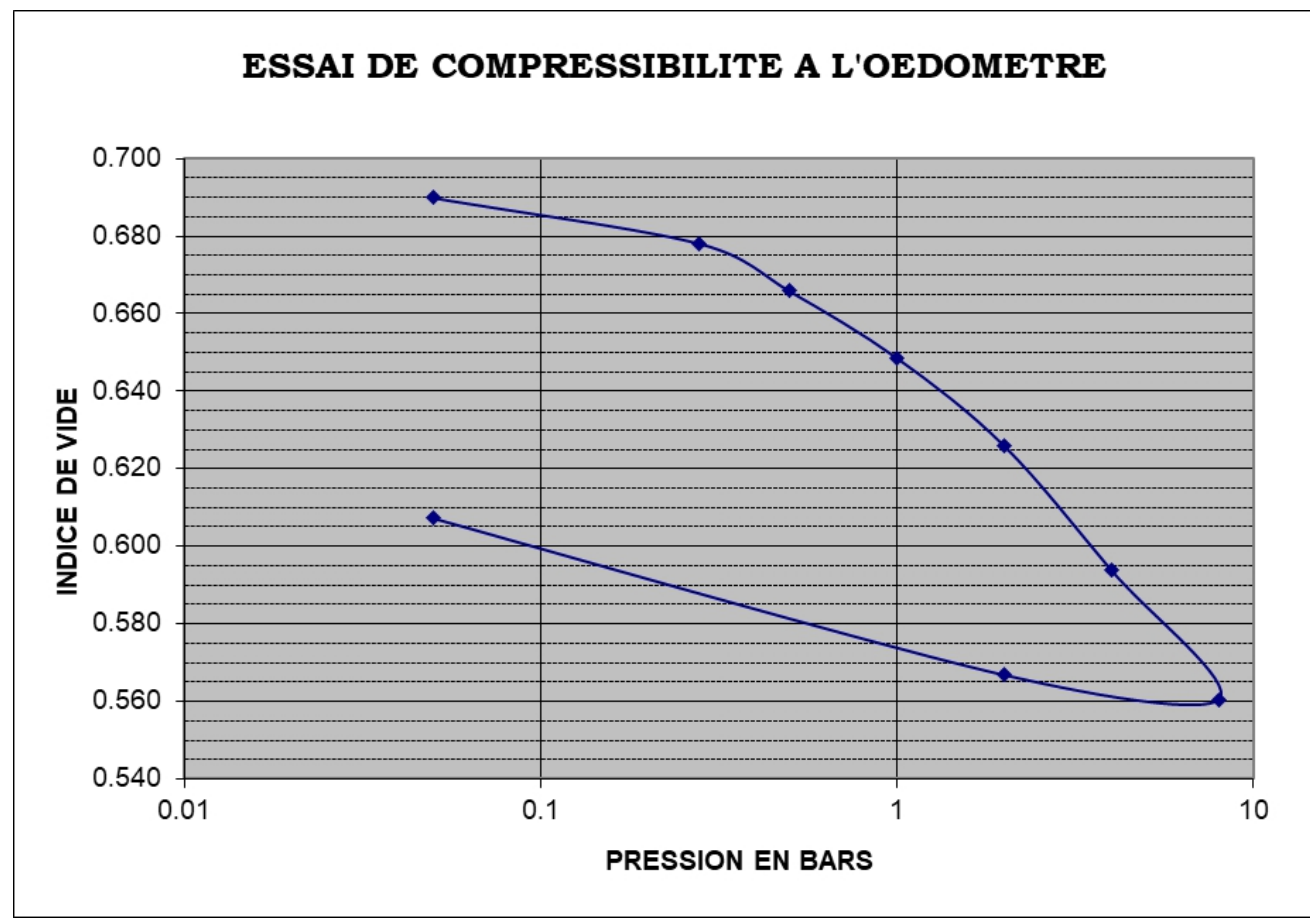

Figure 14: Courbe de compressibilité à l'œdomètre en $\mathrm{P} 2$.

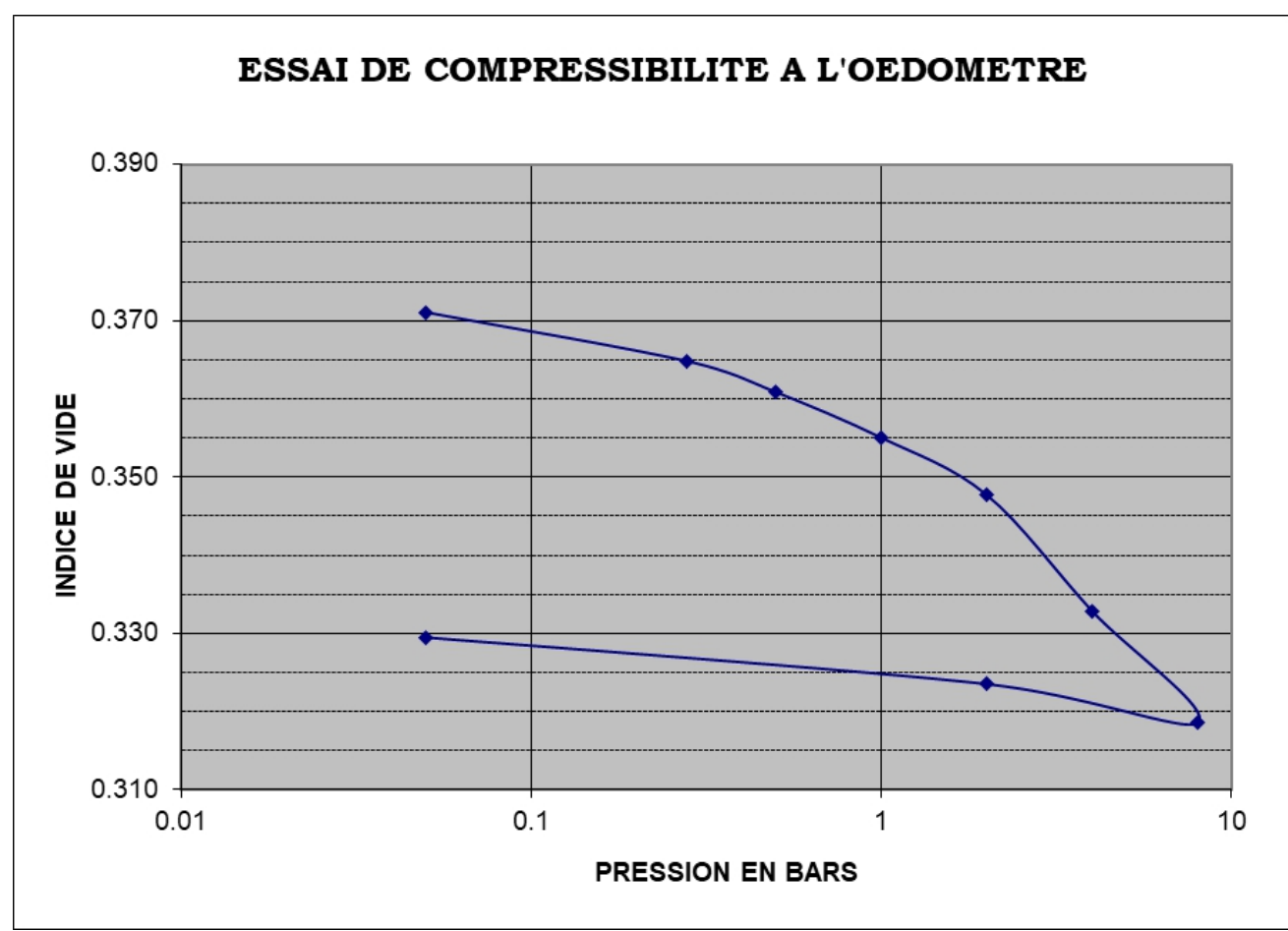

Figure 15: courbe de compressibilité à l'œdomètre en P3. 
Ces courbes permettent de déduire le coefficient de gonflement $\mathrm{C}_{\mathrm{s}}$, le coefficient de compressibilité $\mathrm{C}_{\mathrm{c}}$, et l'indice de gonflement $\mathrm{C}_{\mathrm{r}}$ (tableau5). Les courbes obtenues ont également permis de calculer la plus grande contrainte effective de préconsolidation $\sigma_{c}^{\prime}$. Ces différents paramètres permettent le calcul de tassements $\Delta \mathrm{H}$ dans l'hypothèse d'une uniformité de la couche d'argile à $2 \mathrm{~m}$.

Tableau 5: caractéristiques œdométriques et tassements.

\begin{tabular}{|c|c|c|c|c|c|c|c|c|}
\hline $\begin{array}{l}\mathrm{N}^{\circ} \\
\text { Puits }\end{array}$ & $\begin{array}{c}\text { Indice des } \\
\text { vides } \\
\text { initial }\end{array}$ & $\begin{array}{c}\text { Teneur } \\
\text { en eau } \\
(\% \mathrm{à}\end{array}$ & $\begin{array}{c}\text { Contrainte } \\
\text { initiale } \\
\sigma_{\text {v0 }}^{\prime} \text { (bars) }\end{array}$ & $\mathrm{C}_{\mathrm{c}}$ & $\mathrm{C}_{\mathrm{s}}$ & $\mathrm{C}_{\mathrm{r}}$ & $\sigma_{\mathrm{c}}^{\prime}($ bars $)$ & $\begin{array}{c}\Delta \mathrm{H} \\
\text { En cm. }\end{array}$ \\
\hline P1 & 0,610 & 15,38 & 0,05 & 0,1963 & 0,02 & 0,0235 & 0,3 & 21 \\
\hline P2 & 0,690 & 15,75 & 0,05 & 0,1105 & 0,0159 & 0,0252 & 0,28 & 13 \\
\hline P3 & 0,371 & 15,50 & 0,05 & 0,0475 & 0,0177 & 0,0037 & 0,8 & 6 \\
\hline
\end{tabular}

\section{Discussions}

Les puits manuels font observer que la couche argileuse s'étend audelà de $2,5 \mathrm{~m}$. Les résistances dynamiques ressortent un caractère hétérogène du sol en place, dû vraisemblablement à une variation de compacité. L'échantillon issu du puits $\mathrm{P} 3$ contient des alluvions assez résistantes à la pénétration. A la profondeur de 2 mètres, la valeur de RDP de 124 bars obtenus au point S3 est plus grande que celle obtenue au niveau des deux autres sondages (S1 et $\mathrm{S} 2)$.

L'identification réalisée sur les trois échantillons, montre que l'on est bien en présence des sols fins sensibles à l'eau. Selon le guide du terrassement Routier (GTR, 1992) l'échantillon P1 est de classe A2, l'échantillon P2 est de classe A3 et l'échantillon P3 de classe A1. La différence fondamentale entre les échantillons se situe au niveau de leur plasticité : P1 est assez plastique tandis que P2 est moyennement plastique. L'échantillon P1 est faiblement plastique.

Les essais Proctor donnent des valeurs des poids volumiques secs à l'optimum très proche pour les échantillons $\mathrm{P} 1$ et $\mathrm{P} 2$ respectivement de $1,64 \mathrm{t} / \mathrm{m}^{3}$ et $1,57 \mathrm{t} / \mathrm{m}^{3}$. L'échantillon $\mathrm{P} 3$ se singularise avec un poids volumique sec de $1,88 \mathrm{t} / \mathrm{m}^{3}$ et une teneur en eau optimale $10,48 \%$. Son indice de plasticité est de l'ordre de $11,5 \%$, c'est un matériau alluvionnaire peu plastique confirmant ainsi l'hypothèse selon laquelle l'emplacement échantillonné correspondrait à un dépôt des alluvions transportées par le cours d'eau.

Les courbes de cisaillement obtenu montrent des pics de cisaillement permettant de distinguer clairement l'effort de cisaillement, ce qui montre que ces échantillons sont issus des argiles probablement surconsolidés. Partant des recommandations sur les caractéristiques de cisaillements $\mathrm{Cu}$ et $\varphi_{u}$ (Degoutte et al, 2002) qui doivent être telles que la cohésion soit entre 3 et $30 \mathrm{KPa}$ et l'angle de frottement interne entre $15^{\circ}$ et $40^{\circ}$, les essais réalisées montrent que les échantillons rentrent dans ces fourchettes. De plus on trouve que le plus 
petit coefficient de sécurité au glissement calculé vaut environ 9,28, celui de renversement est 5,04. Ceci montre que l'ouvrage est stable. L'ancrage à $4 \mathrm{~m}$ de la fondation de l'évacuateur de crue résout le problème d'instabilité mécanique. Cependant le déversoir doit être vérifié vis-à-vis des problèmes de tassements. En effet les essais de compressibilité confirment le caractère argileux des échantillons $\mathrm{P} 1$ et $\mathrm{P} 2$ et confirment le caractère identique à celui d'une argile raide kaolinitique. Par contre le sol issu de P3, sa compressibilité de 0,0475 le classe dans les sols sableux argileux.

Le calcul de tassement à $4 \mathrm{~m}$ de profondeur a donné environ $21 \mathrm{~cm}$ au point $\mathrm{P} 1,13 \mathrm{~cm}$ au point $\mathrm{P} 2$ et $6 \mathrm{~cm}$ au point $\mathrm{P} 3$. Entre les différentes positions des échantillons, des tassements différentiels de l'ordre de $8 \mathrm{~cm}$ sont à craindre car l'ouvrage est en béton armé. Ceci impliquerait qu'entre les joints de dilatation proposés dans la structure de l'ouvrage chaque 5 mètres, la distorsion peut atteindre 0,02 (critères de Skempton, et Sowers cas des murs en béton armé). L'ouvrage est en situation de fissuration en permanence, ce qui justifierait probablement l'apparition des fissures avant même la mise en eau de l'ouvrage.

\section{Conclusion et recommandation}

L'étude a conduit à la mesure des paramètres d'identification du sol de fondation constitué des argiles partiellement saturées en P1 et P2 mais une tendance vers un mélange d'un sable argileux obtenu au point P3. Ces données géotechniques imposent que l'ouvrage soit surveillé en permanence, particulièrement vis-à-vis de problèmes de fissurations liés aux tassements du sol. Ceci suppose la mise en place d'une instrumentation avec du matériel de suivi de la déformation. Des visites régulières doivent être conduites afin de réaliser sur des travaux de réparation à temps.

\section{References:}

1. ABN. (2017). Alkongui Data. Niamey (Niger).

2. BABAYE A. (2012, Décembre). Evaluation des ressources en eau souterraine dans le bassin du Dargol (Liptako-Nigerien). Thèse de Doctorat. UAM et Université de Liège.

3. BEN-NAVARETTE M.A. (2009). Mesures dynamiques lors du battage du pénétromètre PANDA2-Génie des procédés. Thèse de Doctorat. Université Blaise Pascal-Clermont Ferrand

4. COMITE FRANÇAIS DES BARRAGES ET RESERVOIRS (CFBR). (2015). Recommandations pour la justification des barrages et digues en remblai. Paris: Editions CFBR-2016.Rue de Clichy, 93584 SaintOuen Cedex - Mise à jour 09/0902 
5. DEGOUTTE G. et al. (1997, 2002). Petits barrages: recommandations pour la conception, la construction et le suivi. Editions CemagrefENGREF, $173 \mathrm{p}$.

6. GAVAUD Y. (1967). Etude des sols du bassin et des alluvions du Gorouol-Beli. ORSTOM

7. GEORGES F \& CAMELAN J.C. Analyses et essais des sols en laboratoire, Conservatoire national des arts et métiers -Paris - Cours de mecaniques des sols.

8. GTR (1992), Realisation des remblais et de couches de formes. Fascicule I-principes généraux SETRA-LCPC.

9. LCPC (1987) Méthode d'essai N²1-Essai de cisaillement à la boîte Diffusion IFSTTAR.

10. Ministère de l'Agriculture. (2012). Etude de réhabilitation du barrage de Youmban. Niamey (Niger): Projet d'appui à la sécurité alimentaire dans les régions de Tillabéry et Dosso (PASA2).

11. NF P94-056. (1996, mars) Sols : Reconnaissances et Essais. Analyse granulométrique. Méthode par tamisage à sec après lavage. AFNOR $16 \mathrm{p}$.

12. NF 94-051. (1993, mars). Sols: Reconnaissances et Essais. Détermination des limites d'Atterberg-limite de liquidité à la coupellelimite de plasticité au Rouleau. AFNOR.

13. NF P94-093. (1997) Essai Proctor Normal-Essai Proctor Modifié. AFNOR $17 \mathrm{p}$.

14. NF P94-071-1 (1994) Sols : Reconnaissances et Essais - Essai de cisaillement rectiligne à la boîte - Partie 1 : Cisaillement direct. AFNOR

15. NF P94-115 (1990) Géotechnique - Sols : Reconnaissances et Essais Essai de pénétration dynamique type B. AFNOR

16. RECA. (2004). Zonage agro-écologique du Niger, mise en oeuvre SDR. Niamey, Niger. Comité interministeriel de Pilotage.

17. REICHEILT R. (1971). Etude géologique resumée du Gourma un "seuil" et un bassin du précambrien supérieur. Thèse de doctorat. Université Clermont Ferrand.

18. ORSTOM. (1970). Etude hydrologique sur la rivière BELI. Burkina Faso.

19. OUSMANE B. (1988). Etude Géochimique et Isotopique des aquifères du socle de la bande sahelienne du Liptako, Sud Maradi, Zinder Est. Niamey: Thèse de doctorat. Université de Niamey. 